\title{
Comparison of Methods to Assess Similarity between Phrases ${ }^{\star}$
}

\author{
Renzo Angles, Valeria Araya, Jesus Concha, and Rodrigo Paredes \\ Department of Computer Science, Universidad de Talca, Chile \\ rangles@utalca.cl, econcha@utalca.cl, raparede@utalca.cl, \\ valeria.araya.m@gmail.com
}

\begin{abstract}
We study the problem of similarity between phrases. To do so, we study three similarity methods. The first one considers the commonalities and differences of the two phrases. The second one is an extension of the well-known Levenshtein-Damerau distance in a word oriented fashion. The third one considers the sequentiality of the phrases and is resistant to phrases with repeated words. Finally, we show an experimental evaluation of our methods in both English and Spanish corpora.
\end{abstract}

Keywords: Phrase similarity, Levenshtein Distance, Word oriented methods.

\section{Introduction}

A phrase is a sequence of consecutive words. The notion of phrase similarity has been studied in several application domains, including document retrieval [1], web document clustering [34] and automatic machine translations [510].

We study phrase similarity in the perspective of improving the quality of automatic translations. Our study is motivated by the deficiencies of current techniques to accurately translate a text from a source to a target language. For example, Table 1 shows the results of translating the phrase "the trip was busy" from English to Spanish, by using four translation services. Considering that the expected result is "el viaje estuvo ocupado", we see that none of them was able to provide the correct translation.

Refining a machine translation implies to modify the translation under a given criteria in order to improve its quality. In the above example, the translated phrase "el viaje fue ocupado" (returned by Google Translator) can be refined by replacing the word "fue" by "estuvo". Our approach for refining is based on exploiting the Web as a huge source of phrases. The idea is building a database of (likely) well written phrases obtained from the Web. Then, given a translated phrase, we will try to find a well written phrase in the database which can be used to refine the input phrase.

In order to solve this problem, we need to define a metric to measure the similarity between phrases. That is, given two phrases, the metric returns a

* This work is partially funded by Fondecyt grant 1131044, Chile.

E. Bayro-Corrochano and E. Hancock (Eds.): CIARP 2014, LNCS 8827, pp. 255-263 2014.

(C) Springer International Publishing Switzerland 2014 
Table 1. Examples of translations, English-Spanish, for the phrase "the trip was busy"

\begin{tabular}{|c|c|}
\hline Translation Service & Result \\
\hline Google Translator (translate.google.com/) & el viaje fue ocupado \\
\hline Bing Translator (www.bing.com/translator) & el viaje estaba ocupado \\
\hline Babylon (traductor.babylon.com/) & el viaje estaba ocupado \\
\hline El Mundo Traductor (www.elmundo.es/traductor/) & el viaje estaba ocupado \\
\hline
\end{tabular}

score which determines how similar the two phrases are (the higher the score the more similarity). Hence, each phrase in the database can be compared with the input phrase and ranked according to its score. The phrase with the highest score is the solution to the problem.

Contributions. This paper presents new contributions to the problem of computing similarity between phrases. First, we formalize a basic notion of similarity found in the literature [8] which is based on the commonalities and differences between two phrases. Second, we extend the Levenshtein distance [7] to compare phrases by considering word-oriented edit operations. Third, we present a sequential method to calculate the cost of a phrase-to-phrase transformation which is simpler than Levenshteins's but preserves the precision. Four, we evaluate and compare the effectiveness and efficiency of the methods in terms of their precision, recall, coverage, sensitivity and execution time.

\subsection{Related Work}

The notion of similarity between phrases has been studied in several application domains, including machine translation [10 2] and phrase-based document similarity [34]. To best of our knowledge, there exist no work concerning the study and comparison of metrics to measure the similarity between phrases. The closest work is the one presented by Lin [8] in 1994. In this article, Lin presents an information-theoretic definition of similarity and demonstrate how to use it to measure similarity. Unfortunately, it is oriented to words comparison.

The Levenshtein distance (also called edit distance) is a discrete function that, given two strings, measures the minimum number of insertions, deletions and substitutions of single characters needed to transform one string into the other. Its plain, recursive implementation requires $O\left(3^{n}\right)$ time in the size of the strings; however, it is easy to obtain a $O\left(n^{2}\right)$ dynamic programming version.

The idea of extending the Levenshtein distance to compute the similarity between phrases has been explored by some authors. Leusch et al [6] defined a measure, called the inversion edit distance, which is based on measuring clocks reordering (i.e., two sentences are similar if a block of words just changes its position). In 2004, Vilares et al [9] presented an algorithm that combines the edit distance between parse trees and single-term similarity. 


\section{Similarity Methods}

In this section we describe three metrics for measuring phrase similarity. For the sake of space economy we omit the pseudocode of the corresponding algorithms. However, they are available in ing . utalca.cl/ rangles/mt/pseudocodes . pdf.

\subsection{Basic Metric}

Consider the intuitive definition of similarity presented in [8]: "The similarity between two objects A and B is a function of their commonalities and differences". This notion of similarity is formalized in the following definition.

Definition 1 (Simple similarity between phrases). The similarity between two phrases, $f_{1}$ and $f_{2}$, is defined as $\operatorname{sim}\left(f_{1}, f_{2}\right)=C-(D+I)$ where $C$ is the number of coincident words, $D$ is the number of words to eliminate, and $I$ is the number of words to insert, all operations required to transform $f_{1}$ into $f_{2}$.

This definition yields to a very obvious algorithm to compare two phrases. Despite its simplicity, it reflects well the intuition that two phrases having several words in common (even if the word are permuted) are more similar than those having several different words (i.e. we need to delete or insert words to transform a phrase into the other).

\subsection{Word-Oriented Edit Distance}

Our second alternative is a rather sophisticated one, inspired on the edit distance. Since a phrase is composed by words, the comparison of two phrases must be based on the words they contain instead of the characters. So, based on the standard version, we define a word-oriented edit distance.

The proposed method considers five types of edit operations: the three basic ones (namely, insertions, deletions or substitutions of single words), the transposition of two adjacent words, and another operation that allows to move a word within a phrase. The latter operation softens the comparison of two phrases that share words in different order. To do so, instead of use the same penalization for every edit operation, we define two values: we penalize with $c I D S$ for insertions, deletions and substitutions of single words, and with $c M T$ for single word movements and transpositions of two adjacent words. After some preliminary experimental evaluation we set $c I D S=8$ and $c M T=2$, as with these values we reflect the intuitive notion of similarity among phrases.

To compute the cost of transforming the phrase $f_{1}=w_{11} w_{12} \ldots w_{1 n}$ into phrase $f_{2}=w_{21} w_{22} \ldots w_{2 m}$, our algorithm takes into account the cost of transforming each of the $n$ prefixes of $f_{1}$ into each of the $m$ prefixes of $f_{2}$. Using a matrix cost of size $(m+1) \times(n+1)$ to manage all the costs of prefix transformation, we can implement an efficient dynamic programming version.

This measure begins by computing size of each phrase $n \leftarrow\left|f_{1}\right|$ and $m \leftarrow\left|f_{2}\right|$. Next, it creates the matrix cost of size $m+1 \times n+1$. In order to minimize the 
cost of transforming the prefix $f_{1,1 \ldots j}=w_{11} w_{12} \ldots w_{1 j}$ into prefix $f_{2,1 \ldots i}=$ $w_{21} w_{22} \ldots w_{2 i}$, we consider several cases: $(i)$ transform prefix $f_{1,1 \ldots j}$ into prefix $f_{2,1 . i-1}$ and pay the cost of insert word $w_{2 i}$; $(i i)$ transform prefix $f_{1,1 \ldots j-1}$ into prefix $f_{2,1 . . i}$ and pay the cost of delete word $w_{1 j}$; $(i i i)$ transform prefix $f_{1,1 \ldots j-1}$ into prefix $f_{2,1 . . i-1}$, and substitute $w_{1 j}$ by $w_{2 i}$ if they differ or do nothing if they are equal; and $(i v)$ transform prefix $f_{1,1 \ldots j-2}$ into prefix $f_{2,1 . . i-2}$ and pay the cost of swapping words $w_{1(j-1)}$ and $w_{1 j}$. Furthermore, in the third case, if the words differ, but the word $w_{2 i}$ belongs to $f_{1}$ we account this as a word movement. As we want to minimize, we pick the minimum cost among insertions, deletions, substitutions, movements and word transpositions.

To initialize the matrix cost we note that the first row represents the cost of transforming $f_{1}$ into the empty phrase, by deleting each of its words. Analogously, the first column represents the cost of transforming the empty phrase in $f_{2}$ by inserting its words. The final result is stored in cell $\operatorname{cost}_{m, n}$.

\subsection{Sequential Distance}

One of the drawbacks we detected in the word-oriented edit distance occurs when there are repetitions of words in the target phrase (phrase $f_{2}$ ). So we devise a sequential approach to compare two phrases.

The basic idea is to traverse both phrases simultaneously, word-wise from the beginning and perform edit operations as needed. If the $i$-th word of both phrases $f_{1}$ and $f_{2}$ coincide, that is, if $w_{1 i}=w_{2 i}$, we advance to the next word. Otherwise, we need to determine whether we need to insert, delete or transpose a word. If word $w_{1 i}$ does not belong to the suffix of $f_{2}$ (which is denoted by $\left.f_{2, i \ldots m}\right)$, we delete $w_{1 i}$ from $f_{1}$ and pay for the cost of deleting it $(c I D)$; else, if $w_{1 i}$ does belong, we preserve it to future process. On the other hand, if word $w_{2 i}$ belongs to the suffix of $f_{1}$ we just fetch it by a circular shifting of the words in the sub-phrase of $f_{1}$ limited by $i$ and the position of $w_{2 i}$ within the suffix of $f_{1}$ (and pay $c T$ for the transposition). Finally, if $w_{2 i}$ does not belong, we need to insert it into $f_{1}$ (and pay $c I D$ for the insertion). Next, we process the next word until consume one or both phrases.

After this processing, it is possible that any of the phrases still has words to process. If so, either all the unprocessed words in $f_{2}$ are inserted into $f_{1}$ and we pay $c I D$ for each insertion; or all the unprocessed words in $f_{1}$ are deleted and pay $c I D$ for each deletion. Finally, we return the dissimilarity assessment.

After some preliminary experimental evaluation, we set $c I D=8$ and $c T=2$, as with these values we reflect the intuitive notion of similarity among phrases.

\section{Experimental Evaluation}

In this section we evaluate the metrics presented above in terms of their effectiveness and efficiency. Recall that effectiveness is purely a measure of the ability of a system to satisfy the user in terms of the relevance of items (words, phrases, documents, etc.) retrieved, whereas efficiency measures the computer resources used by a system to complete the retrieval process. 
As usual in the area of Information Retrieval, the effectiveness of a metric is measured in terms of precision and recall. In our context, the precision of a metric is the fraction of retrieved phrases that are relevant, while recall is the fraction of relevant phrases that are retrieved. Additionally, we consider the notions of coverage and sensitivity. The coverage of a metric is the number of results needed to recover all the relevant phrases. The sensitivity measures the degradation in the recovering capacity of a metric under specific types of errors (swap, insertion or deletion). Finally, the efficiency is the execution time required to rank all the phrases in the corpus according to a given metric. In what follows, we formally define the experiments and discuss the results.

\subsection{Experiments}

In order to define the experiments we carry out in this paper, consider the following general definitions:

- Assume that $C_{L}$ denotes a corpus of phrases in a given language $L$. In this sense, we consider a corpus $C_{e n}$ of English phrases (approx. $48.7 \mathrm{MB}$ ) and a corpus $C_{e s}$ of Spanish phrases (approx. 55.4 MB). Each corpus contains 1 million phrases obtained from DBpedia dumps (wiki.dbpedia.org/Downloads) corresponding to Wikipedia articles.

- For each corpus $C$, we generate a set of 100 test phrases obtained randomly from $C$ (without duplicates).

- Given a test phrase $f$, we generate 10 phrases from $f$ via different transforming operations, specifically: 1 or 2 swaps, 1 or 2 inserts, 1 or 2 deletes, swap + insert, insert + delete, and delete + swap. Note that, the resulting phrases can be considered relevant as they are obtained from the original test phrase.

- Given two phrases $f$ and $f^{\prime}$, a metric is a function $M\left(f, f^{\prime}\right)$ which returns a value representing the similarity between $f$ and $f^{\prime}$. We use $M_{1}$ to denote the basic metric (Section 2.1), $M_{2}$ for the adapted edit-distance (Section 2.2), and $M_{3}$ for the sequential metric (Section 2.3).

- Given a metric $M$, a corpus $C$ and a test phrase $f \in C$, the function $\operatorname{Rank}(M, C, f)$ returns the phrases in $C$ ranked by their similarity with $f$, i.e. each phrase $f^{\prime} \in C$ is ranked according to $M\left(f, f^{\prime}\right)$. The closer to the top in the rank that a phrase $f^{\prime}$ occurs, the more similar is $f^{\prime}$ to the test phrase $f$.

- Given a sorted list of phrases $L$, the function $\operatorname{Top}_{N}(L)$ returns the first $N$ phrases in $L$.

Given a metric $M$, a corpus of phrases $C$, a test phrase $f \in C$ and a total number of phrases to retrieve $k$, we consider the following experiments:

Experiment 1: Calculating Precision. The precision of a metric $M$ is given by the fraction (or percentage) of relevant phrases retrieved by $M$ with respect to the total number of phrases retrieved $k$. Specifically, if $s$ is the number of relevant phrases occurring in $\operatorname{Top}_{k}(\operatorname{Rank}(M, C, f))$ then the precision of $M$ is $s / k$. This 
experiment considers to compute, for each metric $M_{i}$ and corpus $C_{L}$, the average precision over a set of 100 test phrases obtained from $C_{L}$. The precision results are showed in Figures $1(\mathrm{a})$ and 2(a).

Experiment 2: Calculating Recall. The recall of a metric $M$ is given by the fraction (or percentage) of relevant phrases retrieved by $M$ with respect to the total number of relevant phrases. Specifically, if $r$ is the total number of relevant phrases and $s$ is the number of relevant phrases occurring in $\operatorname{Top}_{k}(\operatorname{Rank}(M, C, f))$ then the recall of $M$ is $s / r$. This experiment considers to compute, for each metric $M_{i}$ and corpus $C_{L}$, the average recall over the set of 100 test phrases obtained from $C_{L}$. Figures $1(\mathrm{~b})$ and 2(b) show the recall results.

Experiment 3: Calculating Coverage. The coverage of a metric $M$ is defined as the number (or percentage) of phrases that we need to recover from $\operatorname{Rank}(M, C, f)$ in order to obtain all the relevant phrases. The coverage was calculated with a upper bound of 100 recovered phrases. This experiment considers to compute, for each metric $M_{i}$ and corpus $C_{L}$, the average coverage over the set of 100 test phrases obtained from $C_{L}$.

Experiment 4: Calculating Sensitivity. The sensitivity of a metric $M$ under a type of error $\xi$ (swap, insert or delete operation) measures how the efficiency of the metric is affected by the occurrence of multiple errors of type $\xi$. In this sense, the sensitivity shows the degradation rate of $M$ due to an increasing number of errors of type $\xi$.

Given a test phrase $f$ and a type of error $\xi$, we generate "perturbed" phrases from $f$ by introducing multiple errors of type $\xi$ into $f$, incrementally. Specifically, we construct the set of perturbed phrases $\left\{f_{1}, \ldots, f_{n}\right\}$ where $f_{1}$ contains 1 error, $f_{2}$ contains 2 errors, and so on, until completing $n$ errors. The sensitivity of a metric $M$ according to a set of perturbed phrases (as defined above) is calculated as $\left(\operatorname{Rank}\left(M, C, f_{n}\right)-\operatorname{Rank}\left(M, C, f_{1}\right)\right) /(n-1)$ where $n$ indicates the maximum number of errors. In our experiments, the sensitivity is calculated with $n=5$.

This experiment considers to compute, for each metric $M_{i}$, corpus $C_{L}$ and type of error $\xi$ (swap, insert or delete), the average sensitivity over the set of 100 test phrases obtained from $C_{L}$. The sensitivity results are showed in Figures $1(\mathrm{c})-(\mathrm{e})$ and $2(\mathrm{c})-(\mathrm{e})$

\subsection{Results and Comments}

Figures 1 and 2 show the results of the experimental evaluation for both English and Spanish, respectively.

In terms of precision, Figures 1(a) and 2(a) show that all the methods are very similar. Considering that the total number of relevant phrases is 10 , the precision decreases when the number of phrases retrieved increases from $k=10$ to $k=20$. Specifically, for $k=5$ the precision is almost $100 \%$, for $k=10$ the precision is higher that $85 \%$, and for $k=20$ the precision is mildly lower than $50 \%$. Additionally, we can see that the precisions of $M_{1}$ and $M_{3}$ are very similar whereas $M_{2}$ is slightly lower. 


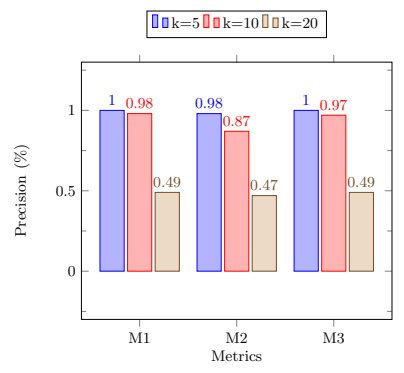

(a) Precision of the metrics.

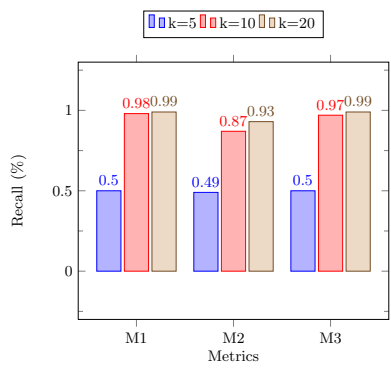

(b) Recall of the metrics.

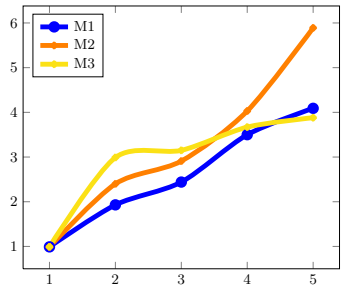

(c) Sensitivity of the metrics under swaps.

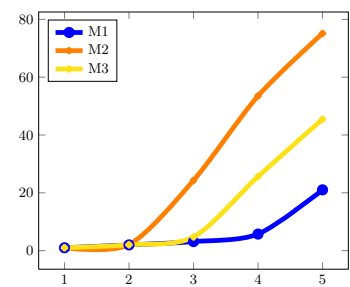

(d) Sensitivity of the metrics under insertions.

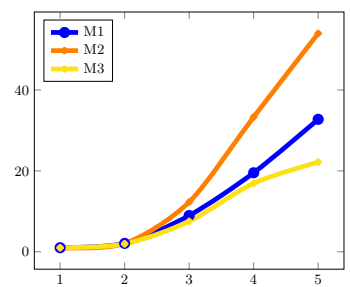

(e) Sensitivity of the metrics under deletions.

Fig. 1. Experimental evaluation over English corpus. $M_{1}$ identifies the basic metric, $M_{2}$ the adapted edit-distance and $M_{3}$ the sequential metric. The total number of relevant phrases is 20 and $k$ defines the number of phrases retrieved.

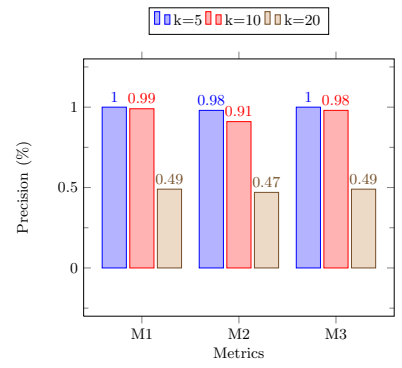

(a) Precision of the metrics.

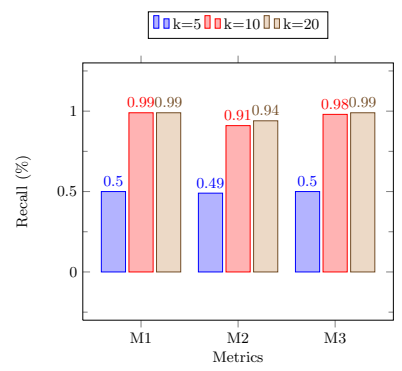

(b) Recall of the metrics.

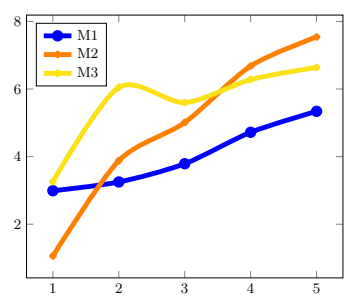

(c) Sensitivity of the metrics under swaps.

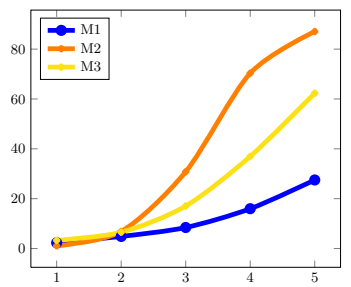

(d) Sensitivity of the metrics under insertions.

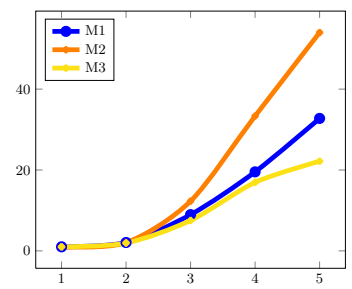

(e) Sensitivity of the metrics under deletions.

Fig. 2. Experimental evaluation over Spanish corpus 
In Figures 1(b) and 2(b), we can see that the three metrics are very similar in terms of recall. For $k=5$, all the metrics are able to recover the $50 \%$ of the total set of relevant phrases, and for $k>=10$ the recall is higher than $85 \%$.

With respect to their coverage, we can indicate (the plot is omitted for lack of space) that the three metrics need less or equal than 20 results to recover the set of 10 relevant phrases. Specifically, $M_{1}$ requires 11 results, $M_{2}$ requires 20 results, and $M_{3}$ requires 10 results. In this sense, $M_{2}$ requires the double of results to recover all the relevant phrases.

Figures 1(c) - (e) and 2(c) - (e) show different behaviors with respect to the sensitivity to swap, insert and delete errors. $M_{1}$ is the least sensitive to swaps and insertions, and $M_{3}$ is the least one to deletions. In contrast, $M_{2}$ is the most sensitive to the three operations. Note that, the values returned by the sensitivity tests are not included, however the degradation rate is visible in the charts.

Finally, we can indicate that the average execution time (in microseconds) is $14 \mu \mathrm{s}$ for $M_{1}, 53 \mu \mathrm{s}$ for $M_{2}$ and $9 \mu \mathrm{s}$ for $M_{3}$, considering phrases in English. In the case of Spanish phrases, the times are $23 \mu \mathrm{s}$ for $M_{1}, 71 \mu \mathrm{s}$ for $M_{2}$, and $19 \mu \mathrm{s}$ for $M_{3}$. Hence, we have that the sequential metric is the most efficient, closely followed by the basic metric, and the adapted edit-distance is the one with the highest average execution time.

\section{Conclusions}

Finally, we will try to answer the question: Which is the best metric? Considering that there is not a unique and clear definition of similarity between phrases, and that the notion of relevance is very subjective, we conclude that there exist no metric that can be efficient and effective for all the cases, when only considering simple edit word operations. However, from the experiments presented in this paper, we can conclude that a simple metric (as the basic metric defined here) can be more effective and efficient than a complex one (as the word-oriented edit-distance) considering the reduced setup of edit operations.

On the other hand, we hope that by introducing semantic elements in the metrics (such as the use of synonyms or semantic similarity measures between words) or by considering the context of the phrases, we can improve the similarity assessment between the phrases.

\section{References}

1. Balasubramanian, N., Allan, J., Croft, W.B.: A comparison of sentence retrieval techniques. In: Proc. 30th ACM SIGIR, pp. 813-814. ACM (2007)

2. Chiang, D.: Hierarchical phrase-based translation. Computational Linguistics 33(2), 201-228 (2007)

3. Hammouda, K.M., Kamel, M.S.: Phrase-based document similarity based on an index graph model. In: Proc. 2002 IEEE ICDM, pp. 203-210 (2002)

4. Hammouda, K.M., Kamel, M.S.: Efficient phrase-based document indexing for web document clustering. IEEE Trans. Knowl. Data Eng. 16(10), 1279-1296 (2004) 
5. Koehn, P., Och, F.J., Marcu, D.: Statistical phrase-based translation. In: Proc. 2003 NAACL, vol. 1, pp. 48-54. Assoc. for Computational Linguistics (2003)

6. Leusch, G., Ueffing, N., Ney, H.: A novel string-to-string distance measure with applications to machine translation evaluation. In: MT Summit IX (2003)

7. Levenshtein, V.I.: Binary codes capable of correcting deletions, insertions, and reversals. Soviet Physics Doklady 10(8), 707-710 (1966)

8. Lin, D.: An information-theoretic definition of similarity. In: Proc. 15th Intl. Conf. on Machine Learning, pp. 296-304. Morgan Kaufmann (1998)

9. Vilares, M., Ribadas, F.J., Vilares, J.: Phrase similarity through the edit distance. In: Galindo, F., Takizawa, M., Traunmüller, R. (eds.) DEXA 2004. LNCS, vol. 3180, pp. 306-317. Springer, Heidelberg (2004)

10. Zens, R., Och, F.J., Ney, H.: Phrase-based statistical machine translation. In: Jarke, M., Koehler, J., Lakemeyer, G. (eds.) KI 2002. LNCS (LNAI), vol. 2479, pp. 18-32. Springer, Heidelberg (2002) 